\title{
Estimates of electronic coupling for excess electron transfer in DNA
}

\author{
Alexander A. Voityuk ${ }^{\text {a) }}$ \\ Institució Catalana de Recerca i Estudis Avançats (ICREA), Institute of Computational Chemistry, \\ Universitat de Girona, 17071 Girona, Spain
}

(Received 20 April 2005; accepted 27 May 2005; published online 22 July 2005)

\begin{abstract}
Electronic coupling $V_{d a}$ is one of the key parameters that determine the rate of charge transfer through DNA. While there have been several computational studies of $V_{d a}$ for hole transfer, estimates of electronic couplings for excess electron transfer (ET) in DNA remain unavailable. In the paper, an efficient strategy is established for calculating the ET matrix elements between base pairs in a $\pi$ stack. Two approaches are considered. First, we employ the diabatic-state (DS) method in which donor and acceptor are represented with radical anions of the canonical base pairs adenine-thymine (AT) and guanine-cytosine (GC). In this approach, similar values of $V_{d a}$ are obtained with the standard $6-31 G^{*}$ and extended $6-31++G^{* *}$ basis sets. Second, the electronic couplings are derived from lowest unoccupied molecular orbitals (LUMOs) of neutral systems by using the generalized Mulliken-Hush or fragment charge methods. Because the radical-anion states of AT and GC are well reproduced by LUMOs of the neutral base pairs calculated without diffuse functions, the estimated values of $V_{d a}$ are in good agreement with the couplings obtained for radical-anion states using the DS method. However, when the calculation of a neutral stack is carried out with diffuse functions, LUMOs of the system exhibit the dipole-bound character and cannot be used for estimating electronic couplings. Our calculations suggest that the ET matrix elements $V_{d a}$ for models containing intrastrand thymine and cytosine bases are essentially larger than the couplings in complexes with interstrand pyrimidine bases. The matrix elements for excess electron transfer are found to be considerably smaller than the corresponding values for hole transfer and to be very responsive to structural changes in a DNA stack. (C) 2005 American Institute of Physics. [DOI: 10.1063/1.1961400]
\end{abstract}

\section{INTRODUCTION}

There are two types of charge transfer in DNA: (1) positive charge or electron-hole transfer (HT) when a radicalcation state moves from one base pair to another and (2) excess electron transfer (ET) when a radical-anion state migrates through the $\pi$ stack. The majority of experimental and theoretical studies of charge transfer in DNA have been related to electron-hole migration, i.e., to the propagation of radical-cation states of nucleobases along the duplex. Recently it has been shown that excess electron transfer has a large potential for nanodevices, ${ }^{1}$ in particular, for DNA chip technology. ${ }^{2}$ In the past, investigations of excess electron transport were restricted to electron-spin resonance. ${ }^{3}$ However, several photochemical and spectroscopic studies have been published recently ${ }^{4-7}$ (see also references in a review ${ }^{8}$ ). Therefore, computational insight into the mechanistic and dynamic issues of excess electron transport in DNA is now of special interest. Elementary steps for charge transfer in DNA were considered by Berlin et al. ${ }^{9}$ General aspects of charge-transfer modeling in DNA have recently been considered in an excellent review. ${ }^{10}$

The electronic coupling of donor and acceptor $V_{d a}$ is a key characteristic which controls the rate of charge transfer and mainly determines its sensitivity to the arrangement of donor and acceptor. There are several studies on estimation

${ }^{a)}$ Electronic mail: alexander.voityuk@icrea.es and analysis of electronic couplings for hole transfer in DNA. ${ }^{11,12}$ Quantum-chemical schemes to compute the electronic matrix element in DNA and their applications have been recently considered. ${ }^{13}$

The ability of DNA to mediate an excess electron is associated with the formation of radical-anion states of nucleobases. There exist two types of radical-anion states of nucleobases: (i) dipole-bound anions where the excess electron is located far outside the molecule and (ii) valence (covalently bound) anionic state the electron is delocalized over the molecule. The existence of dipole-bound anions was first predicted theoretically by Adamowicz and co-workers ${ }^{14,15}$ and subsequently detected in the gas-phase experiments by Desfrançois et al. ${ }^{16}$ and Hendricks et al. ${ }^{17,18}$ In the gas phase, the nucleobase anions may be described as electron-dipolebound states. ${ }^{19-21}$ However, it has been shown that hydrogen bonds between an anion and its surroundings stabilize the valence-bound state. ${ }^{19-21}$ For instance, a computational study of the adenine-thymine (AT) Watson-Crick pair predicted the formation of the covalent anion with the positive vertical electron detachment energy. ${ }^{19}$ The energetics and structure of radical-anion states of nucleobases and their complexes have been intensively studied theoretically. ${ }^{22-27}$ Unlike positively charged systems considered for HT, the quantum-mechanical treatment of negatively charged models employed for exploring ET is more complicated, ${ }^{21-27}$ in particular, a considerable influence of the basis set on calculation results is expected.

Thus far, no estimates of electronic couplings are avail- 
able for excess electron transfer in DNA. In this work, we establish an efficient strategy for calculating these matrix elements.

\section{COMPUTATIONAL DETAILS}

\section{A. Electronic coupling}

We estimated electronic couplings using two approaches. First, we invoked a technique for the direct estimation of $V_{d a}$ from the predetermined diabatic states (DSs). ${ }^{28}$ In this scheme, the electronic coupling between diabatic states $\psi_{d}$ and $\psi_{a}$ of the donor and acceptor is defined as

$$
V_{d a}=\frac{H_{d a}-S_{d a}\left(H_{d d}+H_{a a}\right) / 2}{1-S_{d a}^{2}} .
$$

The wave functions $\psi_{d}$ and $\psi_{a}$ are calculated for separated donor and acceptor. In one-electron approximation, these states are represented by Hartree-Fock orbitals. For ET through DNA stack, $\psi_{d}$ and $\psi_{a}$ may be approximated with highest occupied molecular orbital (HOMO) of radical-anion base pairs. The quantities $H_{d d}, H_{a d}$, and $H_{d a}$ as well as the overlap integrals $S_{d a}$ are calculated using the corresponding matrix elements computed in atomic-orbital (AO) basis for the whole system.

Second, the generalized Mulliken-Hush (GMH) scheme $^{29}$ and the fragment charge method ${ }^{30}(\mathrm{FCM})$ were applied to derive ET coupling matrix elements from the results of Hartree-Fock calculations of neutral DNA stacks. All electronic structure calculations were carried out with the standard $6-31 \mathrm{G}^{*}$ and extended $6-31++\mathrm{G}^{* *}$ basis sets using the GAUSSIAN 03 program. ${ }^{31}$ For open-shell systems, the unrestricted Hartree-Fock method was used. Molecular-orbital plots were generated with the help of the program MOLDEN. ${ }^{32}$

\section{B. Geometry of dimers}

The following scheme used previously ${ }^{12}$ was applied to construct dimers consisting of two Watson-Crick pairs. Geometries of the systems were produced using the program SCHNARP. ${ }^{33}$ The relative positions of the nucleobases in these duplexes correspond to the regular DNA structure; the distance between the stacks and the twist between consecutive pairs were assumed to be $3.38 \AA$ and $36^{\circ}$, respectively. The experimental geometries of nucleobases were employed. ${ }^{34}$ While the structure of nucleobases deforms upon trapping of an excess electron, ${ }^{25-27}$ this effect was not accounted for in the present calculations.

\section{RESULTS AND DISCUSSION}

\section{A. Diabatic states}

In a stack consisting of two base pairs, the diabatic donor and acceptor states for excess electron transfer can be approximated by radical-anion states of the separated base pairs. In one-electron approximation, the wave function of an excess electron may be described by HOMO of the radical anion. Figure 1 compares HOMOs of an AT radical anion calculated with the standard and extended basis sets. As seen, both calculations provide very similar results; the excess
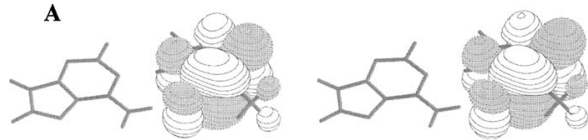

B

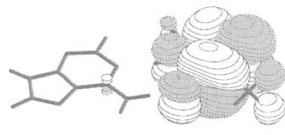

$6-31 G^{*}$

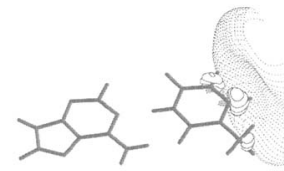

$6-31++G^{* *}$
FIG. 1. (A) HOMO of the AT radical anion and (B) LUMO of the neutral AT base pair. The Hartree-Fock calculations were performed with the standard $6-31 G^{*}$ (in the left) and extended $6-31++G^{* * *}$ (in the right) basis sets.

electron is completely localized on thymine and the wave functions describe a covalently bound anion. A state of the excess electron in the AT anion can also be approximated by lowest unoccupied molecular orbital (LUMO) of the neutral AT base pair. As shown in Fig. 1(B), the LUMO wave function depends dramatically on the basis set employed in the calculation. When diffuse functions are explicitly excluded (6-31G ${ }^{*}$ basis), LUMO of the neutral pair closely resembles HOMO of the AT anion [Fig. 1(A)]. Therefore, this LUMO can be used to describe the state of an excess electron. By contrast, the LUMO calculated with diffuse functions (6-31 $++G^{* *}$ basis) predicts a dipole-bound state for the excess electron and substantially differs from HOMO of the radical anion. This qualitative analysis suggests that the diabatic states for electron transfer may be represented by LUMOs of the neutral $\pi$ stack only when the diffuse functions are explicitly excluded from the basis set used in the calculation.

Similar results were also obtained for a guanine-cytosine (GC) base pair, which forms a covalently bound anion with the excess electron localized on cytosine. Our Hartree-Fock data on the charge distribution in AT and GC anions are in good agreement with the DFT study of Richardson et al. ${ }^{25,26}$ It was found that the AT and GC radical anions form covalently bound states in which the excess charge is localized on pyrimidine bases. ${ }^{25,26}$

Recently, Li and Sevilla have shown that DFT calculations employing basis sets without diffuse functions produce reasonable estimates of the relative valence electron affinities. ${ }^{23}$ Furthermore they found that inclusion of diffuse functions in the basis sets may lead to contamination of the valence state with the dipole-bound state and thereby deteriorate the estimates. ${ }^{23}$ It is well known that extended basis sets supplemented with diffuse functions are required to properly describe molecular anions. However, the calculation with the standard basis sets can be served as a useful "practical" tool for dealing with radical-anion states of nucleobases. ${ }^{23}$ Therefore, it appears to be interesting to test how reliable are electronic coupling calculated with the standard basis sets.

Analysis of molecular orbitals (Fig. 1) supports this approach. Indeed, using the standard and extended basis set we obtain very similar wave functions of an excess electron in AT and GC radical anions; moreover, LUMO and LUMO +1 of the stack [(AT), (AT)] calculated with the standard basis sets [Fig. 2(A)] describe covalently bound anion states 

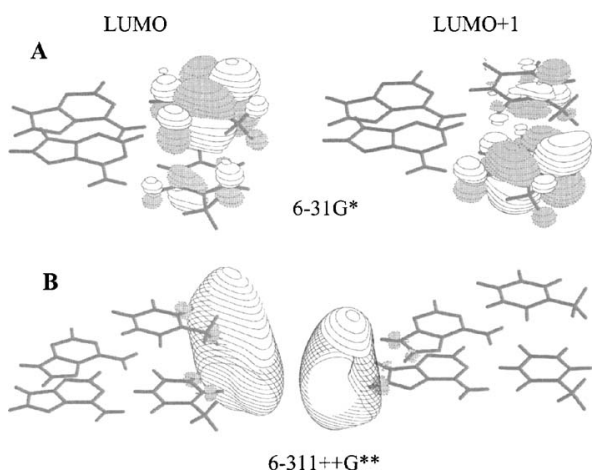

FIG. 2. LUMO and LUMO +1 of the neutral AT base pair calculated with different basis sets: (A) HF/6-31G ${ }^{*}$ calculation and (B) $\mathrm{HF} / 6-31++\mathrm{G}^{* *}$ calculation.

delocalized over thymine bases. These MOs can be presented as linear combinations of the orbitals of an excess electron in the AT pair (Fig. 1). However, employing the extended basis set we find LUMOs which represent the dipole-bound states with a diffuse charge distribution [see Fig. 2(B)] around thymines (LUMO) and adenines (LUMO+1). It is apparent that these wave functions cannot be employed to derive the coupling of valence anionic states. Calculations with diffuse functions $\left(\mathrm{HF} / 6-31++\mathrm{G}^{* *}\right)$ predict that in DNA stacks an excess electron forms covalently bound radical anions with the charge almost completely confined to pyrimidine bases. Thus, even in isolated $\pi$ stacks, the diabatic states for excess electron transfer are covalently bound. Obviously, accounting for the polar environment of DNA will further stabilize valence anion states and suppress the formation of diffuse electron-dipole states. Therefore, we can infer that the diabatic states for excess electron transfer in DNA are well described without diffuse functions. In Sec. III B, we justify this assumption by comparing ET couplings estimated using different computational schemes.

\section{B. Calculation of electronic couplings}

First, we calculate the matrix element $V_{d a}$ for several complexes of base pairs employing the DS method. This scheme has been already used to compute electronic couplings for hole transfer in DNA. ${ }^{35,36}$ As discussed above, the electron donor and acceptor states can be described by HOMO wave functions calculated for the AT and GC radical anions.

In systems $[(\mathrm{AT}),(\mathrm{AT})],[(\mathrm{GC}),(\mathrm{AT})]$, and $[(\mathrm{GC}),(\mathrm{GC})]$, the donor and acceptor sites for the excess electron, pyrimidine bases $\mathrm{T}$ and $\mathrm{C}$, are on the same strand. In Table I, we compare the $V_{d a}$ values calculated with the standard $6-31 G^{*}$ and extended $6-31++G^{* *}$ basis sets. The matrix element in $[(\mathrm{AT}),(\mathrm{AT})]$ is remarkably smaller, $\sim 0.03 \mathrm{eV}$, than that found in $[(\mathrm{GC}),(\mathrm{AT})]$ and $[(\mathrm{GC}),(\mathrm{GC})], \sim 0.10 \mathrm{eV}$. The coupling values depend on the basis sets employed in the calculation; exclusion of diffuse functions leads to some decrease of the coupling in $[(\mathrm{AT}),(\mathrm{AT})]$, while the matrix elements become larger in $[(\mathrm{GC}),(\mathrm{AT})]$ and $[(\mathrm{GC}),(\mathrm{GC})]$. As we will see later, small conformational fluctuations in a $\pi$
TABLE I. Electronic couplings of AT and GC base pairs for excess electron transfer as calculated by the DS method for anion-radical states and by GMH and FCM for neutral systems (in eV).

\begin{tabular}{lcccc}
\hline \hline & \multicolumn{2}{c}{ DS method } & GMH & \multicolumn{2}{c}{ FCM } \\
\cline { 2 - 3 } Complex & $6-31++\mathrm{G}^{* *}$ & $6-31 \mathrm{G}^{*}$ & \multicolumn{2}{c}{$6-31 \mathrm{G}^{*}$} \\
\hline$[(\mathrm{AT}),(\mathrm{AT})]$ & 0.034 & 0.025 & 0.029 & 0.030 \\
{$[(\mathrm{GC}),(\mathrm{AT})]$} & 0.066 & 0.113 & 0.107 & 0.107 \\
{$[(\mathrm{GC}),(\mathrm{GC})]$} & 0.078 & 0.1092 & 0.119 & 0.119 \\
{$[(\mathrm{AT}),(\mathrm{TA})]$} & 0.022 & 0.018 & 0.023 & 0.023 \\
{$[(\mathrm{GC}),(\mathrm{TA})]$} & 0.019 & 0.017 & 0.019 & 0.018 \\
{$[(\mathrm{GC}),(\mathrm{CG})]$} & 0.016 & 0.017 & 0.019 & 0.019 \\
\hline \hline
\end{tabular}

stack may cause changes in $V_{d a}$ which are considerably larger than the difference in the matrix elements calculated with the standard and extended basis sets.

In the stacks $[(\mathrm{AT}),(\mathrm{TA})][(\mathrm{GC}),(\mathrm{TA})][(\mathrm{GC}),(\mathrm{CG})]$, the $\mathrm{T}$ and $\mathrm{C}$ bases are on opposite strands. In these systems, the couplings of interstrand pyrimidine bases are essentially smaller than intrastrand matrix elements just considered. The interstrand couplings are about $0.02 \mathrm{eV}$ while the intrastrand matrix elements fall in the range of $0.03-0.1 \mathrm{eV}$. Because the diabatic states for ET in $\pi$ stacks appear to be well approximated by radical-anion states of AT and GC, the couplings estimated by the DS method may be used as reference values.

The matrix elements $V_{d a}$ were also obtained with the GMH and FCM schemes (Table I). For these calculations we employed LUMO and LUMO+1 orbitals calculated for neutral stacks with HF/6-31G* . As seen from Table I, the GMH and FCM results are in good agreement with the DS values. Thus, the GMH and FCM diabatization schemes provide very similar $V_{d a}$ estimates when applied to virtual electronic states of donor and acceptor calculated without diffuse functions. We explicitly note that including diffuse functions leads to completely wrong $V_{d a}$ estimates based on virtual states for neutral complexes.

It has been shown that HT couplings are very sensitive to conformational changes in DNA stacks. ${ }^{35,37}$ One expects that also ET couplings should be responsive to the structural variations. As an example, let us consider a complex [(AT), (AT)]. The dependence of $V_{d a}$ on the system geometry is demonstrated by the data collected in Table II. We chose the increments of the base-step parameters (Fig. 3) in line with their standard deviations extracted from x-ray data. ${ }^{38}$ More details on the generation of distorted structures can be found elsewhere. ${ }^{37}$ The $V_{d a}$ matrix element between the AT pairs is very responsive to conformational changes; its values range from 0.003 to about $0.06 \mathrm{eV}$ (Table II). The coupling rises significantly as the distance between donor and acceptor decreases, from $0.003 \mathrm{eV}$ at rise $=3.88 \AA$ to $0.058 \mathrm{eV}$ at rise $=2.88 \AA$. Variation of the twist angle (rotation around the DNA axis) from $31^{\circ}$ to $41^{\circ}$ leads to the decrease of the matrix element by a factor of 2 . Thus, ET matrix elements are very responsive to structural fluctuations as are HT couplings, ${ }^{35,37}$ and this effect has to be accounted for by modeling of excess charge migration in DNA. The average value of ET couplings presented in Table II is about 
TABLE II. Electronic coupling matrix element $\left|V_{d a}\right|$ for excess electron transfer in different conformations of $[(\mathrm{AT}),(\mathrm{AT})]$ as calculated by GMH and FCM (in eV).

\begin{tabular}{|c|c|c|c|}
\hline \multicolumn{2}{|c|}{ Conformation } & \multirow{2}{*}{$\begin{array}{c}\text { GMH } \\
0.022\end{array}$} & \multirow{2}{*}{$\begin{array}{l}\text { FCM } \\
0.023\end{array}$} \\
\hline & & & \\
\hline \multirow[t]{2}{*}{ Rise $(\AA)$} & 2.88 & 0.057 & 0.058 \\
\hline & 3.88 & 0.003 & 0.003 \\
\hline \multirow[t]{2}{*}{ Shift $(\AA)$} & 0.5 & 0.017 & 0.016 \\
\hline & -0.5 & 0.047 & 0.047 \\
\hline \multirow[t]{2}{*}{ Slide $(\AA)$} & 1 & 0.054 & 0.054 \\
\hline & -1 & 0.029 & 0.028 \\
\hline \multirow[t]{2}{*}{ Roll } & $5^{\circ}$ & 0.026 & 0.027 \\
\hline & -5 & 0.021 & 0.021 \\
\hline \multirow[t]{2}{*}{ Tilt } & $2^{\circ}$ & 0.027 & 0.028 \\
\hline & $-2^{\circ}$ & 0.018 & 0.019 \\
\hline \multirow[t]{2}{*}{ Twist } & $31^{\circ}$ & 0.024 & 0.024 \\
\hline & $41^{\circ}$ & 0.013 & 0.013 \\
\hline
\end{tabular}

$\overline{\bar{a} \text { In the reference conformation, rise }=3.38 \AA \text {, twist }=36^{\circ} \text {, and other base-step }}$ parameters are zero.

$0.027 \mathrm{eV}$. It is essentially smaller than the corresponding average value calculated for the HT coupling in [(AT), (AT)], $0.078 \mathrm{eV} .^{37}$ ET electronic couplings are expected to be smaller than the corresponding HT matrix elements because for any system the LUMO has one node more than the HOMO. Respectively, the overlap of diabatic states for ET should be smaller than for $\mathrm{HT}$, and therefore, $\left|V_{d a}(\mathrm{ET})\right|$ $<\left|V_{d a}(\mathrm{HT})\right|$. In some special stack conformations, one may find the opposite relation between couplings, $\left|V_{d a}(\mathrm{ET})\right|$ $>\left|V_{d a}(\mathrm{HT})\right|$.

\section{CONCLUSIONS}

Electronic couplings $V_{d a}$ have been estimated for excess electron transfer in DNA $\pi$ stacks consisting of two base pairs. Complexes with intra- and interstrand donor and acceptor sites have been considered. Two different methods were employed. The direct calculations were performed using diabatic states of donor and acceptor represented by radical-anion states of separated base pairs. Electronic structure calculations of the AT and GC base pairs and their complexes were carried out using the Hartree-Fock method with the standard $6-31 \mathrm{G}^{*}$ and extended $6-31++\mathrm{G}^{* * *}$ basis sets. The matrix elements $V_{d a}$ calculated with the small and extended basis sets are found to be in good agreement. We also showed that electronic couplings for excess electron transfer can be reasonably well estimated using LUMO and LUMO

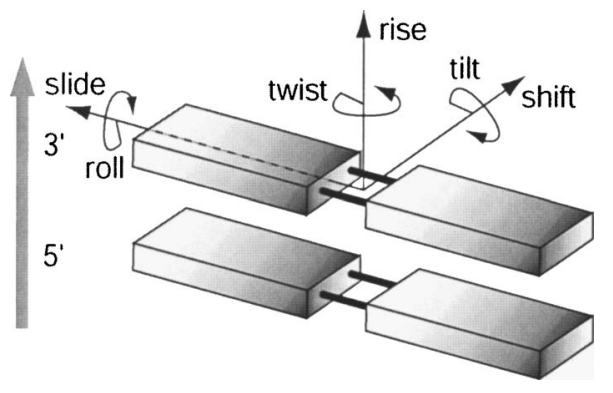

FIG. 3. The six base-step parameters that define the relative position of two nearest base pairs in a DNA stack.
+1 of neutral DNA $\pi$ stacks. Within this approach, however, calculations have to be performed without diffuse functions, for instance, using the standard $6-31 \mathrm{G}^{*}$ basis. If so the excess electron is confined to the molecular framework and the diabatic states represented by LUMOs of neutral base pairs closely resemble the radical-anion states calculated with extended basis sets. The generalized Mulliken-Hush and fragment charge methods produce very similar estimates of $V_{d a}$ which are in good agreement with values found with the diabatic-state approach. Thus, the HF/6-31G* calculations of neutral $\pi$ stacks of base pairs allow for reasonable estimates of electronic coupling for excess electron transfer. However, physically unreasonable values of the couplings will be derived from the calculations of neutral stacks with basis sets augmented by diffuse functions. On average, the ET couplings are found to be essentially smaller than the corresponding HT matrix elements. Similar to $V_{d a}$ for hole transfer, the ET matrix elements are shown to be very sensitive to conformational changes of $\pi$ stacks.

This study demonstrated that the electronic couplings for excess electron transfer in DNA can be reasonably estimated in a similar manner that is used for calculating the hole transfer-matrix elements. Because relative energetics for ET can also be derived from calculations without diffuse functions, ${ }^{23}$ we conclude that modeling of negative charge migration in DNA can be performed using efficient computational procedures that employ small basis sets.

${ }^{1}$ S. J. Park, T. A. Taton, and C. A. Mirkin, Science 295, 1503 (2002).

${ }^{2}$ E. M. Boon, J. E. Salas, and J. K. Barton, Nat. Biotechnol. 20, 282 (2002).

${ }^{3}$ Z. Cai and M. D. Sevilla, in Long-Range Charge Transfer in DNA: Topics in Current Chemistry, edited by G. B. Shuster (Springer, Berlin, 2004), Vol. 237, pp. 103-127.

${ }^{4}$ F. D. Lewis, X. Liu, S. E. Miller, R. T. Hayes, and M. R. Wasielewski, J. Am. Chem. Soc. 124, 11280 (2002).

${ }^{5}$ B. Giese, B. Carl, T. Carl, T. Carell, C. Behrens, U. Hennecke, O. Schiemann, and E. Feresin, Angew. Chem., Int. Ed. 43, 1848 (2004).

${ }^{6}$ T. Ito and S. E. Rokita, Angew. Chem., Int. Ed. 43, 1839 (2004).

${ }^{7}$ P. Kaden, E. Mayer-Enthart, A. Trifonov, T. Fiebig, and H.-A. Wagenknecht, Angew. Chem., Int. Ed. 44, 1636 (2005).

${ }^{8}$ H. A. Wagenknecht, Angew. Chem., Int. Ed. 42, 2454 (2003).

${ }^{9}$ Y. A. Berlin, A. L. Burin, and M. A. Ratner, Chem. Phys. 275, 61 (2002).

${ }^{10}$ Y. A. Berlin, I. V. Kurnikov, D. N. Beratan, M. A. Ratner, and A. L. Burin, in Long-Range Charge Transfer in DNA: Topics in Current Chemistry, edited by G. B. Shuster (Springer, Berlin, 2004), Vol. 237, pp. 1-36.

${ }^{11}$ A. A. Voityuk, N. Rösch, M. Bixon, and J. Jortner, J. Phys. Chem. B 104, 9740 (2000).

${ }^{12}$ A. A. Voityuk, J. Jortner, M. Bixon, and N. Rösch, J. Chem. Phys. 114, 5614 (2001).

${ }^{13}$ N. Rösch and A. A. Voityuk, in Long-Range Charge Transfer in DNA: Topics in Current Chemistry, edited by G. B. Shuster (Springer, Berlin, 2004), Vol. 237, pp. 37-72.

${ }^{14}$ N. A. Oyler and L. Adamowicz, Chem. Phys. Lett. 219, 223 (1994).

${ }^{15}$ L. Adamowicz, J. Phys. Chem. 97, 11122 (1993).

${ }^{16}$ C. Desfrançois, H. Abdoul-Carime, and J. P. Schermann, J. Chem. Phys. 102, 7792 (1996).

${ }^{17}$ J. H. Hendricks, S. A. Lyapustina, H. L. de Clercq, J. T. Snodgrass, and K. H. Bowen, J. Chem. Phys. 104, 7788 (1996).

${ }^{18}$ J. H. Hendricks, S. A. Lyapustina, H. L. de Clercq, and K. H. Bowen, J. Chem. Phys. 108, 8 (1998).

${ }^{19}$ I. Al-Jihan, J. Smets, and L. Adamowicz, J. Phys. Chem. A 104, 2994 (2000).

${ }^{20}$ L. Adamowicz, J. Phys. Chem. 100, 14655 (1996).

${ }^{21}$ S. G. Stepanian, I. O. Reva, E. D. Radchenko, and L. Adamowicz, J. Phys. Chem. A 103, 4404 (1999); D. M. Smith, Y. Elkady, and L. Adamowicz, ibid. 103, 5784 (1999). 
${ }^{22}$ M. D. Sevilla, B. Besler, and A.-O. Colson, J. Phys. Chem. 99, 1060 (1995).

${ }^{23} \mathrm{X}$. Li and M. D. Sevilla, J. Phys. Chem. A 106, 1596 (2002).

${ }^{24}$ N. Russo, M. Toscano, and A. Grand, J. Comput. Chem. 14, 1243 (2000).

${ }^{25}$ N. A. Richardson, S. S. Wesolowski, and H. F. Schaefer III, J. Am. Chem. Soc. 124, 10163 (2002).

${ }^{26}$ N. A. Richardson, S. S. Wesolowski, and H. F. Schaefer III, J. Phys. Chem. B 107, 848 (2003).

${ }^{27}$ M. Haranczyk and M. Gutowski, J. Am. Chem. Soc. 127, 699 (2005).

${ }^{28}$ M. D. Newton, Chem. Rev. (Washington, D.C.) 91, 767 (1991).

${ }^{29}$ R. J. Cave and M. D. Newton, J. Chem. Phys. 106, 9213 (1997).

${ }^{30}$ A. A. Voityuk and N. Rösch, J. Chem. Phys. 117, 5607 (2002).
${ }^{31}$ M. J. Frisch et al., GAUSSIAN 03, Revision B.04, Gaussian, Inc., Pittsburgh, PA, 2003.

${ }^{32}$ G. Schaftenaar and J. H. Noordik, J. Comput.-Aided Mol. Des. 14, 123 (2000).

${ }^{33}$ X. J. Lu, M. A. El Hassan, and C. A. Hunter, J. Mol. Biol. 273, 681 (1997).

${ }^{34}$ L. Clowney, S. C. Jain, A. R. Srinivasan, J. Westbrook, W. K. Olson, and H. W. Berman, J. Am. Chem. Soc. 118, 509 (1996).

${ }^{35}$ A. Troisi and G. Orlandi, J. Phys. Chem. B 106, 2093 (2002).

${ }^{36}$ A. A. Voityuk and N. Rösch, Isr. J. Chem. 44, 109 (2004).

${ }^{37}$ A. A. Voityuk, K. Siriwong, and N. Rösch, Phys. Chem. Chem. Phys. 3, $5431(2001)$

${ }^{38}$ C. A. Hunter and X. J. Lu, J. Mol. Biol. 265, 603 (1997). 